\title{
Serum free sulfhydryl status is associated with patient and graft survival in
}

\section{renal transplant recipients}

Anne-Roos S. Frenay ${ }^{1}$, Martin H. de Borst ${ }^{2}$, Matthias Bachtler ${ }^{3}$, Nadine Tschopp ${ }^{3}$, Charlotte A. Keyzer ${ }^{2}$, Else van den Berg ${ }^{2}$, Stephan J.L. Bakker ${ }^{2,5}$, Martin Feelisch ${ }^{4}$, Andreas Pasch $^{3}$ and Harry van Goor ${ }^{1}$

${ }^{1}$ Pathology and Medical Biology, University Medical Center Groningen and University of Groningen, the Netherlands

${ }^{2}$ Nephrology, University Medical Center Groningen and University of Groningen, the Netherlands

${ }^{3}$ Department of Clinical Research, University of Bern, Inselspital, Switzerland

${ }^{4}$ Clinical and Experimental Sciences, Faculty of Medicine, University of Southampton, Southampton General Hospital, Southampton, United Kingdom

${ }^{5}$ Top Institute Food and Nutrition, Wageningen, The Netherlands

Running title: Free thiols in Kidney transplantation

Word count abstract: 262

Word count abstract and text: 2946

Corresponding author:

Prof. dr. H. van Goor

Department of Pathology and Medical Biology, University Medical Center Groningen and University of Groningen

Hanzeplein 1, 9700 RB Groningen, the Netherlands

Phone: +31 503619529 Fax: +31 503619107 E-mail: h.van.goor@umcg.nl 


\section{Abstract}

Oxidative stress contributes significantly to graft failure, morbidity and mortality in renal transplant recipients (RTR). In cells, free sulfhydryl groups (reduced thiols, R-SH) are the transducers of redoxregulated events; their oxidation status is modulated by interaction with reactive oxygen and nitrogen oxide species and thought to be in equilibrium with the circulating pool. We hypothesized that high levels of serum free thiols are a reflection of a favorable redox status and therefore positively associated with cardiovascular risk parameters, patient and graft survival in RTR.

To test this, reactive free thiol groups (R-SH; corrected for total protein) were quantified in serum of 695 RTR ( $57 \%$ male, $53 \pm 13 \mathrm{yr}$, functioning graft $\geq 1 \mathrm{yr}$ ) using Ellman's Reagent, and R-SH determinants were evaluated with multivariable linear regression models. Associations between R-SH and mortality or graft failure were assessed using multivariable Cox regression analyses.

In multivariable models, male gender, estimated glomerular filtration rate and serum thiosulfate positively associated with R-SH while BMI, HbA1c, corrected calcium and NT-pro-BNP inversely associated with R-SH (model $\mathrm{R}^{2}=0.26$ ). During follow-up (3.1 [2.7-3.9] yrs), 79 (11\%) patients died and 45 (7\%) patients developed graft failure. R-SH correlated inversely with all-cause mortality (HR 0.58 [95\% Cl $0.45-0.75]$ per SD increase) and graft failure (HR 0.42 [0.30 - 0.59]; both $P<0.001$ ), independent of parameters with which R-SH significantly associated in the multivariable regression analyses, except for NT-pro-BNP.

Serum R-SH are associated with a beneficial cardiovascular risk profile and better patient and graft survival in RTR, suggesting potential usefulness as low-cost, high-throughput screening tool for whole-body redox status in translational studies. Whether R-SH modification improves long-term outcome of RTR warrants further exploration.

Keywords: cardiovascular disease - free thiols - graft failure - kidney transplantation - mortality oxidative stress 


\section{Introduction}

Renal transplantation is the preferred treatment for most patients with end-stage renal disease (ESRD). Although both quality of life and prognosis of renal transplant recipients (RTR) have significantly improved ${ }^{1}$, morbidity and mortality rates remain high ${ }^{2}$.

Aberrant production of reactive oxygen species (ROS) causes oxidative stress and is assumed to be one of the hazardous players that contribute to the development of graft failure and patient mortality in RTR. ${ }^{3}$ Via the induction of an increased inflammatory response, oxidative stress leads to the onset and progression of many diseases, including cardiovascular disease (CVD). ${ }^{4}$ High levels of free sulfhydryl groups (R-SH) may be a reflection of a favorable redox status since they avidly interact with ROS and other reactive species. ${ }^{5}$ In healthy individuals, the concentrations of serum protein thiols are highly regulated ${ }^{6}$ and form part of an intricate redox network that underpins human adaptation to nutritional, metabolic and environmental challenges. ${ }^{7}$ Conditions promoting oxidative stress result in the oxidation of free sulfhydryl groups to their corresponding disulfides, which is associated with risk for CVD. ${ }^{6}$ The extracellular redox status influences CVD through proinflammatory signaling, which involves mitochondrial oxidation, nuclear factor- $\mathrm{KB}$ activation and elevated expression of genes for monocyte recruitment to endothelial cells. ${ }^{6}$ While the thiol/disulfide status of glutathione and cysteine, for example, are acknowledged readouts of oxidative stress their analytical determination at the prevailing low concentrations in plasma or serum can be challenging and requires specific detection techniques such as high-pressure liquid chromatography or mass spectrometry not available to all laboratories. Moreover, at present there is no consensus about the significance of specific low-molecular-weight or protein thiols for disease protection and many low-abundant thiols are prone to rapid artificial oxidation. Less timeconsuming, more robust yet affordable alternatives requiring only a spectrophotometer or plate reader would therefore be of value for potential use in translational medicine, but examples in the literature are few and many studies are underpowered. Based on the associations of free sulfhydryl groups with oxidative stress, we hypothesized that total free sulfhydryl groups might have merit as a gross read-out of cardiovascular health in RTR. In the present study we therefore investigated whether reactive sulfhydryl groups in serum are associated with a beneficial cardiovascular risk profile (a.o. NT-pro-BNP) and improved patient and graft survival in stable RTR. To this end, we measured total free sulfhydryl status using Ellman's Reagent in serum of 695 stable RTR and analyzed its relationship with cardiovascular risk parameters, graft failure and all-cause mortality. 


\section{Subjects and Methods}

\section{Study design and population}

The study protocol was approved by the Institutional Review Board (METc 2008/186) and was in adherence to the Declaration of Helsinki. From November 2008 till June 2011, we invited all stable RTR ( $\geq 18$ years, $n=817$ ) with a functioning graft for over one year, which were treated in the outpatient clinic of the University Medical Center Groningen (UMCG), the Netherlands. After giving written informed consent, a total of 707 (87\%) participated in the present study. R-SH was measured in serum samples of 695 RTR (98\%). Further details of the study population have been published previously. ${ }^{8,9}$

\section{Outcome parameters}

The primary outcome measures of this study were all-cause mortality and the death-censored graft failure. The latter was defined as restart of dialysis or retransplantation. Outcome measures were recorded until the end of May 2013; median follow-up was 3.1 [2.7 - 3.9] years, with no participants lost to follow-up.

\section{Clinical parameters}

Information on participants' health status, medical history and medication use was extracted from patient records. To categorize smoking behavior into current, former or never smoked, a self-report questionnaire was used. Relevant transplant information was extracted from the UMCG renal transplant database. Blood pressure and heart rate were measured using a semi-automatic device (Dinamap 1846, Critikon, Tampa, FL, USA) every minute for the duration of fifteen minutes, following a strict protocol. ${ }^{10}$ An average of the last three values was taken as a final value. Body weight and height were measured and body mass index (BMI) was calculated as weight divided by height squared $\left(\mathrm{kg} / \mathrm{m}^{2}\right)$. As described previously ${ }^{8}$, all participants were instructed to collect $24 \mathrm{~h}$-urine sample at the day prior to their visit to the outpatient clinic. In the morning after an overnight fasting period and completing the $24 \mathrm{~h}$-urine collection, blood was drawn and subsequently venous blood gas analyses were performed photometrically. Electrolytes, phosphate, albumin, urea and creatinine in plasma and urine were measured using routine laboratory methods, which was also the case for serum cholesterol, HbA1c and hs-CRP. Urinary sulfate and thiosulfate concentrations were measured as previously described. ${ }^{8}$ Serum thiosulfate concentrations were determined by HPLC following delipidation by 2 chloroform-methanol (2:1) extraction steps and derivatization of thiosulfate with $18.4 \mathrm{mM}$ mono-bromobimane. ${ }^{11}$ Samples were measured using a Waters Alliance 2695 HPLC, LiChrospher 60 RP select B (Merck Milipore) columns and a RF-10AXL fluorescence detector 
(Shimadzu, Kyoto, Japan). Mobile phase was a $0.25 \%$ acetic acid in deionized $\mathrm{H}_{2} \mathrm{O} /$ methanol gradient (88:12 to 0:100, both Merck Milipore).

Renal function was assessed by calculating the estimated glomerular filtration rate (eGFR) using the CKD Epidemiology Collaboration (CKD-EPI) equation. ${ }^{12}$ Serum calcium was corrected for hypoalbuminemia $(<40 \mathrm{~g} / \mathrm{L})$ using the following formula: corrected calcium = serum calcium $(\mathrm{mmol} / \mathrm{L})$ $+0.02 \times(40-$ serum albumin $[\mathrm{g} / \mathrm{L}])$.

To determine protein carbonylation, serum protein was precipitated with $20 \%$ trichloroacetic acid before derivatization with 2,4 dinitrophenylhydrazine (both Sigma Aldrich, Buchs, Switzerland, DNPH CAS-number 119-26-6) and spectrophotometric measurement of the resultant (orange) reaction products at $400 \mathrm{~nm}$ (reference $650 \mathrm{~nm}$ ), as described by Levine and colleagues. ${ }^{13}$

\section{R-SH measurement; colorimetric detection of free thiol groups}

Serum samples were stored at $-80^{\circ} \mathrm{C}$ until R-SH measurement. Free thiol groups were detected as described previously ${ }^{14,15}$, with slight modifications. In short, serum samples were diluted 1:4 with $0.1 \mathrm{M}$ Tris buffer ( $\mathrm{pH} 8.2$ ). After measuring background absorption at $412 \mathrm{~nm}$ using a Sunrise microplate reader (Tecan Group AG, Männedorf, Switzerland), with a reference measurement at 630 nm, $10 \mu \mathrm{L} 3.8$ mM 5,5'-Dithio-bis(2-nitrobenzoic acid) (DTNB; Ellman's Reagent, CAS-number 69-783, Sigma Aldrich Corporation, St. Louis, MO, USA) in 0.1M phosphate buffer ( $\mathrm{pH} 7)$ was added to the samples; after an incubation period of 20 minutes at room temperature sample absorbances were measured again. Concentrations of free thiol groups were determined by parallel measurements of a L-Cysteine (CAS-number 52-90-4, Fluka Biochemika, Buchs, Switzerland) standard curve [15.625 $\mu \mathrm{M}$ to $1000 \mu \mathrm{M}$ ] in $0.1 \mathrm{M}$ Tris/10M EDTA (pH 8.2). The intra- and interday variability of the -SH determinations in the transplant cohort both have a CV $<10 \%$. Finally, to determine the R-SH status per RTR, free thiol groups were corrected for total serum protein, by calculating the free thiol groups/total serum protein ratio. This correction was performed since proteins largely determine the amount of potentially measurable free thiol groups (the total pool of free low-molecular weight thiols is typically below $10 \mu \mathrm{M}$ whereas the total protein RSH pool of healthy individuals is in the hundreds of $\mu \mathrm{M})$.

\section{Statistical analysis}

To test the distribution of all parameters, histograms and probability plots were displayed followed by the Kolmogorov-Smirnov test. Skewed data were normalized for analyses by logarithmic transformation (hsCRP, triglycerides, albuminuria, serum and urinary thiosulfate). The study population was subdivided into tertiles of R-SH to visualize linear and non-linear associations with RSH. To establish $P$-values for differences in R-SH tertiles an ANOVA test was used for normally distributed continuous data, whereas the Kruskal-Wallis test was used for non-normally distributed 
data and the $\chi^{2}$-test for nominal data. To identify the independent determinants of $\mathrm{R}-\mathrm{SH}$, univariable and multivariable linear regression analyses were performed. Multivariable linear regression models were constructed using backward selection $\left(P_{\text {out }}>0.05\right)$, which included all the variables that were significantly associated with R-SH in the univariable analysis.

Tertiles of R-SH were tested for associations with all-cause mortality and death-censored graft failure by Kaplan-Meier analysis, including the log-rank test.

For the Cox regression analyses, models were constructed with inclusion of the potential confounders of R-SH. These are the parameters that significantly associated with R-SH in the multivariable analysis. We first performed crude Cox regression analyses (model 1) and analyses with adjustment for age and gender (model 2). Additionally, eGFR was added (model 3), after which we adjusted for all potential confounders ( $\mathrm{Hb}, \mathrm{HbA1c}$, corrected calcium, hsCRP, serum TS, use of calcineurin inhibitors and use of sirolimus) (model 4). Since storage time showed an inverse association with free thiols value, in all models of the Cox regression analysis we corrected for this parameter.

Furthermore, we evaluated discrimination, which is the ability of the different models to distinguish those who develop an event (i.e. mortality or graft failure) from those who do not develop and event, by calculation of the Harrell C-index for the different models. The Harrell C-index is analogous to the area under the receiver-operating characteristic (ROC) curve and is compatible with time-toevent data. We also calculated the cut-off points for R-SH with optimal sensitivity and specificity, and repeated Cox regression analyses using these cut-off points.

Data are presented as mean \pm standard deviation (SD), unless stated otherwise. A two-sided $P$-value $<0.05$ was considered statistically significant. Statistical analyses were performed using SPSS 22.0 for Windows (SPSS Corp. Chicago, IL, USA) and GraphPad Prism version 5.00 for Windows (GraphPad Software, San Diego, CA, USA). 


\section{Results}

\section{Patient characteristics}

The patient cohort of 695 RTR had a median age of $53.0 \pm 12.8$ years and $57 \%$ were male. The median time between transplantation and the baseline measurement was 5.4 [1.9 - 12.1] years. Serum R-SH was normally distributed with a mean value of $130.89 \pm 49.32 \mu \mathrm{M}$ or $1.83 \pm 0.67$ $\mu \mathrm{M}$ /gram total protein (Figure $1 A$ ). An inverse association of storage time with serum R-SH level was apparent $(p<0.001$ ) (Figure $1 B)$. Baseline characteristics of the RTR are summarized per tertile of R-SH in Table 1. Participants within the highest tertile of R-SH were significantly younger, more likely to be male and with a lower prevalence of diabetes mellitus (DM). Patients in the highest and middle R-SH tertile also had a lower BMI when compared to the lowest R-SH tertile. With regard to the transplantation characteristics, there were no differences between tertiles. Renal function was better in participants within the highest R-SH tertile when compared to the other tertiles. Furthermore, RTR with the highest R-SH also had the highest values of hemoglobin ( $\mathrm{Hb})$ and serum and urinary thiosulfate, and lower $\mathrm{HbA1c}$, corrected serum calcium, serum phosphate, high-sensitive CRP (hsCRP), NT-pro-BNP, total cholesterol, triglycerides and urinary albumin levels. There was no difference in carbonylation between R-SH tertiles. Anti-hypertensives, statins, vitamin $\mathrm{K}$ antagonists and calcineurin inhibitors (CNI) were less often used in participants with high R-SH.

Table 2 reveals parameters that were associated with R-SH in multivariate linear regression analyses: male gender, BMI, HbA1c, renal function, corrected calcium, NT-pro-BNP and serum TS were independently associated with serum R-SH (Table 2 ; model $\mathrm{R}^{2}=0.26$ ). 
Fig 1.

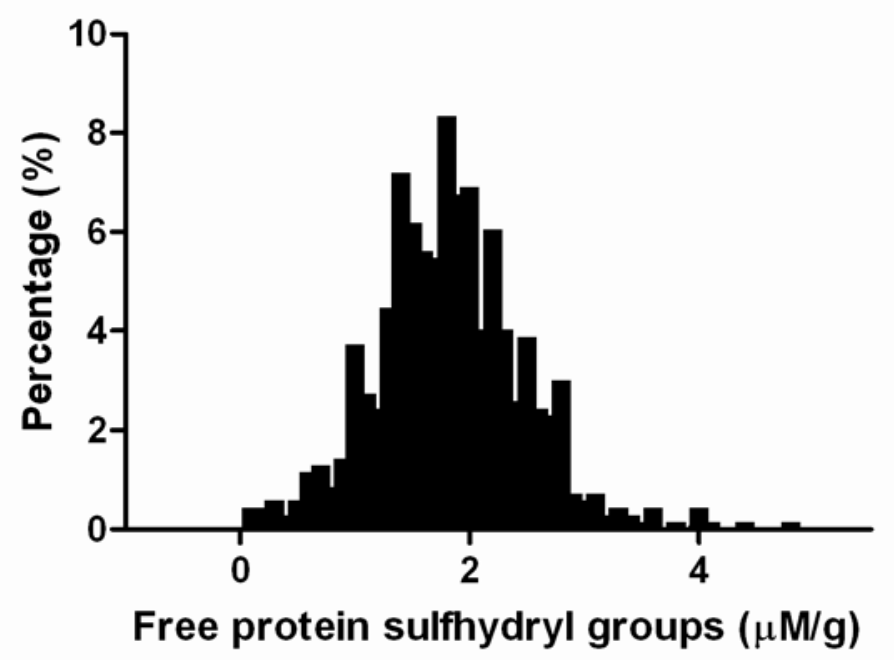

Figure 1A. Histogram of serum R-SH showing a normal distribution curve in RTR

The concentration of serum free thiol groups $(1.83 \pm 0.67 \mu \mathrm{M} / \mathrm{g})$ measured in 695 renal transplant recipients was normally distributed.

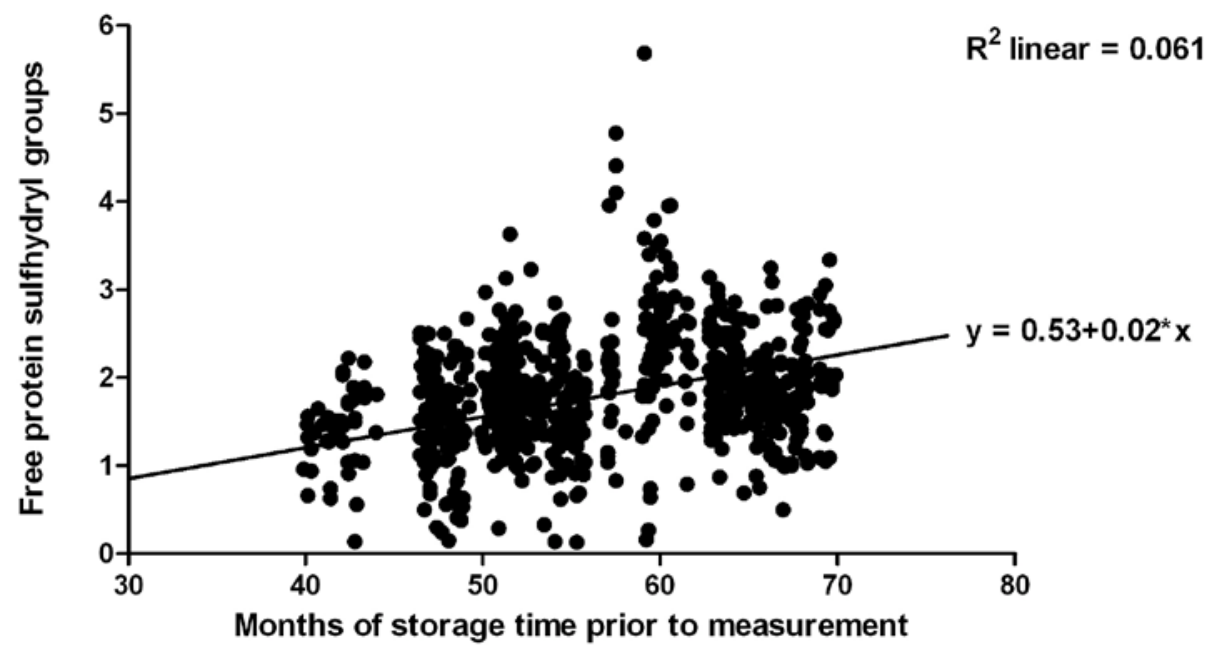

Figure 1B. An inverse association of sample storage time with serum R-SH in RTR

To test whether total serum thiol content was subject to progressive oxidation while kept frozen at $80^{\circ} \mathrm{C}$ individual R-SH levels were plotted against date of sample collection. An inverse relation of duration of storage on serum thiol content was apparent $(p<0.001)$. 
Table 1. Baseline patient characteristics presented as tertiles of R-SH

\begin{tabular}{|c|c|c|c|c|c|}
\hline \multicolumn{6}{|c|}{$\begin{array}{c}\text { Renal Transplant Recipients } \\
\text { Tertiles of R-SH }\end{array}$} \\
\hline & Overall & Tertile 1 & Tertile 2 & Tertile 3 & \multirow{2}{*}{$P$-value } \\
\hline & $N=695$ & $N=233$ & $N=230$ & $N=232$ & \\
\hline R-SH/total protein, $\mu \mathrm{M} / \mathrm{g}$ & $1.83 \pm 0.67$ & $<1.56$ & $1.56-2.04$ & $>2.04$ & $<0.001$ \\
\hline \multicolumn{6}{|l|}{ Demographics } \\
\hline Age, yr & $53.0 \pm 12.8$ & $56.5 \pm 10.8$ & $53.1 \pm 13.0$ & $49.3 \pm 13.5$ & $<0.001$ \\
\hline Male gender, n (\%) & $395(57)$ & $111(48)$ & $127(55)$ & $157(68)$ & $<0.001$ \\
\hline Current smoker, $\mathrm{n}(\%)$ & $84(13)$ & $22(10)$ & $29(13)$ & $33(15)$ & 0.25 \\
\hline Current diabetes, n (\%) & $167(24)$ & $70(30)$ & $50(22)$ & $47(20)$ & 0.03 \\
\hline $\mathrm{BMI}, \mathrm{kg} / \mathrm{m}^{2}$ & $26.6 \pm 4.7$ & $27.6 \pm 5.1$ & $26.0 \pm 4.5$ & $26.2 \pm 4.5$ & 0.001 \\
\hline Mean Arterial Pressure, $\mathrm{mmHg}$ & $100 \pm 12$ & $100 \pm 13$ & $100 \pm 12$ & $101 \pm 11$ & 0.79 \\
\hline Heart rate, bpm & $68.6 \pm 11.9$ & $69.6 \pm 11.9$ & $67.9 \pm 11.7$ & $68.4 \pm 12.1$ & 0.32 \\
\hline \multicolumn{6}{|l|}{ Renal Transplantation } \\
\hline Transplant vintage, years & $5.4[1.9-12.1]$ & $5.6[1.1-15.0]$ & $5.3[1.7-11.9]$ & $5.5[2.9-10.1]$ & 0.85 \\
\hline Living donor, n (\%) & $236(35)$ & $68(29)$ & $80(35)$ & $88(39)$ & 0.09 \\
\hline Pre-emptive KTx, n (\%) & $112(16)$ & $39(17)$ & $35(15)$ & $38(16)$ & 0.90 \\
\hline HLA mismatches, $\mathrm{n}$ & 2 [1-3] & $2[1-3]$ & $2[1-3]$ & 2 [1-3] & 0.23 \\
\hline Age donor, years & $42.8 \pm 15.5$ & $44.3 \pm 15.3$ & $43.2 \pm 15.8$ & $41.0 \pm 15.3$ & 0.07 \\
\hline Acute rejection, n (\%) & $185(27)$ & $61(26)$ & $68(30)$ & $56(24)$ & 0.41 \\
\hline \multicolumn{6}{|l|}{ Laboratory measurements } \\
\hline Haemoglobin, $\mathrm{mmol} / \mathrm{L}$ & $8.2 \pm 1.1$ & $8.0 \pm 1.1$ & $8.2 \pm 1.0$ & $8.5 \pm 1.1$ & $<0.001$ \\
\hline $\mathrm{HbA} 1 \mathrm{C}, \%$ & $5.98 \pm 0.83$ & $6.16 \pm 0.94$ & $5.96 \pm 0.75$ & $5.83 \pm 0.76$ & $<0.001$ \\
\hline $\begin{array}{l}\text { eGFR, CKD-EPI } \\
\left(\mathrm{ml} / \mathrm{min} / 1.73 \mathrm{~m}^{2}\right)\end{array}$ & $52.3 \pm 20.1$ & $43.6 \pm 18.1$ & $53.8 \pm 18.2$ & $59.6 \pm 20.6$ & $<0.001$ \\
\hline Corrected calcium $\mathrm{mmol} / \mathrm{L}$ & $2.34 \pm 0.15$ & $2.37 \pm 0.15$ & $2.34 \pm 0.14$ & $2.31 \pm 0.14$ & $<0.001$ \\
\hline Phosphate, $\mathrm{mmol} / \mathrm{L}$ & $0.96 \pm 0.21$ & $1.02 \pm 0.22$ & $0.95 \pm 0.20$ & $0.92 \pm 0.20$ & $<0.001$ \\
\hline Thiosulfate, $\mu \mathrm{mol} / \mathrm{L}$ & $3.63[2.50-5.26]$ & $3.12[2.05-5.12]$ & $3.50[2.41-4.84]$ & $4.17[3.0-5.65]$ & $<0.001$ \\
\hline Magnesium, mmol/L & $0.96 \pm 0.12$ & $0.96 \pm 0.11$ & $0.95 \pm 0.13$ & $0.95 \pm 0.13$ & 0.37 \\
\hline PTH, pmol/L & 8.9 [5.9-14.7] & $9.8[6.5-16.4]$ & 8.9 [6.1-13.9] & $8.3[5.5-14.0]$ & 0.10 \\
\hline Venous $\mathrm{pH}$ & $7.37 \pm 0.04$ & $7.37 \pm 0.04$ & $7.37 \pm 0.04$ & $7.37 \pm 0.04$ & 0.60 \\
\hline Venous $\mathrm{HCO}_{3}{ }^{-}, \mathrm{mmol} / \mathrm{L}$ & $24.6 \pm 3.1$ & $24.4 \pm 3.2$ & $24.8 \pm 3.0$ & $24.8 \pm 3.0$ & 0.31 \\
\hline hsCRP, $\mathrm{mg} / \mathrm{L}$ & $1.6[0.7-4.5]$ & $1.9[0.9-5.5]$ & $1.6[0.6-4.4]$ & $1.2[0.6-3.1]$ & 0.002 \\
\hline NT-pro-BNP, ng/L & $252[108-634]$ & 439 [190 - 1233] & $247[111-584]$ & $139[59-330]$ & $<0.001$ \\
\hline Alkaline phosphatase, $\mathrm{U} / \mathrm{L}$ & $67[54-83]$ & $69[57-92]$ & $67[54-80]$ & $66[51-81]$ & 0.06 \\
\hline Total cholesterol, $\mathrm{mmol} / \mathrm{L}$ & $5.0[4.4-5.8]$ & $5.3[4.7-6.1]$ & $4.9[4.3-5.7]$ & $4.8[4.3-5.5]$ & $<0.001$ \\
\hline HDL cholesterol, mmol/L & $1.3[1.1-1.6]$ & $1.3[1.1-1.7]$ & $1.3[1.1-1.7]$ & $1.3[1.0-1.6]$ & 0.05 \\
\hline LDL cholesterol, mmol/L & $2.9[2.3-3.5]$ & $3.0[2.4-3.7]$ & $2.9[2.3-3.5]$ & $2.8[2.3-3.4]$ & 0.06 \\
\hline Triglycerides, $\mathrm{mmol} / \mathrm{L}$ & $1.68[1.25-2.30]$ & $1.84[1.30-2.64]$ & $1.56[1.24-2.13]$ & $1.63[1.15-2.22]$ & 0.001 \\
\hline $\begin{array}{l}\text { Carbonylation, } \mathrm{nmol} / \mathrm{mg} \\
\text { protein }\end{array}$ & $0.51[0.30-1.14]$ & $0.54[0.33-0.83]$ & $0.49[0.27-0.75]$ & $0.50[0.29-0.78]$ & 0.15 \\
\hline Albuminuria, mg/24h & $40[11-177]$ & 63 [14-304] & $32[8-114]$ & 29 [9-127] & 0.002 \\
\hline Urinary thiosulfate, $\mu \mathrm{mol} / 24 \mathrm{~h}$ & $7.02[3.95-11.89]$ & $5.75[3.24-9.74]$ & $7.00[3.91-11.92]$ & $8.34[5.08-15.24]$ & $<0.001$ \\
\hline Urinary sulfate, $\mathrm{mmol} / 24$ & $17.55 \pm 6.38$ & $17.14 \pm 6.02$ & $17.91 \pm 6.30$ & $17.58 \pm 6.81$ & 0.43 \\
\hline \multicolumn{6}{|l|}{ Medication } \\
\hline Anti-hypertensives, n (\%) & $612(88)$ & $220(94)$ & $200(87)$ & $192(83)$ & $<0.001$ \\
\hline Statins, $\mathrm{n}(\%)$ & $368(53)$ & $139(60)$ & $121(53)$ & $108(47)$ & 0.024 \\
\hline Calcium supplements, n (\%) & $147(21)$ & $56(24)$ & $43(19)$ & $48(21)$ & 0.36 \\
\hline Vitamin D supplements, $\mathrm{n}(\%)$ & $170(25)$ & $63(27)$ & $56(24)$ & $51(22)$ & 0.45 \\
\hline Vitamin K antagonists, n (\%) & $78(11)$ & $39(17)$ & $24(10)$ & $15(7)$ & 0.002 \\
\hline Prednisone, $\mathrm{mg} / \mathrm{d}(\mathrm{n})$ & $10[7.5-10](687)$ & $10[7.5-10](233)$ & $10[7.5-10](227)$ & $10[7.5-10](227)$ & 0.19 \\
\hline Calcineurin inihibitors, n (\%) & $398(57)$ & $148(64)$ & $143(62)$ & $107(46)$ & $<0.001$ \\
\hline Proliferation inhibitor, n (\%) & $579(83)$ & $183(79)$ & $193(84)$ & $202(87)$ & 0.06 \\
\hline Sirolimus, $\mathrm{n}(\%)$ & $12(2)$ & $7(3)$ & $3(1)$ & $2(1)$ & 0.12 \\
\hline \multicolumn{6}{|c|}{ 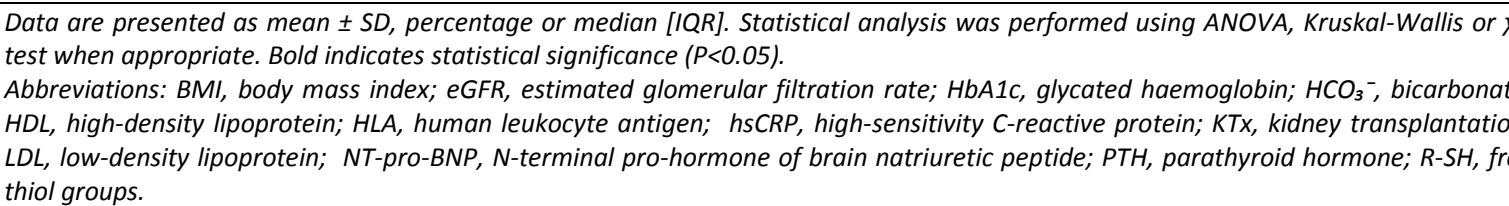 } \\
\hline
\end{tabular}


Table 2. Univariable and multivariable associations of R-SH with clinical parameters in RTR

\begin{tabular}{|c|c|c|c|c|}
\hline \multicolumn{5}{|c|}{ Serum free sulfhydryl groups } \\
\hline & \multicolumn{2}{|c|}{ Univariable } & \multicolumn{2}{|c|}{ Multivariable } \\
\hline & St. Beta & $P$-value & St. Beta & $P$-value \\
\hline \multicolumn{5}{|l|}{ Demographics } \\
\hline Age, yr & -0.211 & $<0.001$ & & \\
\hline Gender (male $=0$ ) & -0.200 & $<0.001$ & -0.114 & 0.005 \\
\hline Current smoker & 0.052 & 0.19 & & \\
\hline Current diabetes & -0.086 & 0.024 & & \\
\hline $\mathrm{BMI}, \mathrm{kg} / \mathrm{m}^{2}$ & -0.131 & 0.001 & -0.084 & 0.04 \\
\hline Mean Arterial Pressure, $\mathrm{mmHg}$ & 0.024 & 0.55 & & \\
\hline Heart rate, bpm & -0.027 & 0.49 & & \\
\hline \multicolumn{5}{|l|}{ Renal Transplantation } \\
\hline Transplant vintage, years & -0.063 & 0.10 & & \\
\hline Living donor & 0.071 & 0.06 & & \\
\hline Pre-emptive $\mathrm{KTx}$ & -0.016 & 0.67 & & \\
\hline HLA mismatches & 0.047 & 0.23 & & \\
\hline Age donor, years & -0.087 & 0.03 & & \\
\hline Acute rejection & -0.032 & 0.40 & & \\
\hline \multicolumn{5}{|l|}{ Laboratory measurements } \\
\hline Hemoglobin, $\mathrm{mmol} / \mathrm{L}$ & 0.250 & $<0.001$ & & \\
\hline $\mathrm{HbA} 1 \mathrm{C}, \%$ & -0.142 & $<0.001$ & -0.102 & 0.01 \\
\hline $\begin{array}{c}\text { eGFR, CKD-EPI } \\
\left(\mathrm{ml} / \mathrm{min} / 1.73 \mathrm{~m}^{2}\right)\end{array}$ & 0.347 & $<0.001$ & 0.143 & 0.002 \\
\hline Corrected calcium mmol/L & -0.141 & $<0.001$ & -0.092 & 0.02 \\
\hline Phosphate, $\mathrm{mmol} / \mathrm{L}$ & -0.212 & $<0.001$ & & \\
\hline Thiosulfate, $\mu \mathrm{mol} / \mathrm{L}$ & 0.110 & 0.004 & 0.095 & 0.01 \\
\hline Magnesium, mmol/L & -0.061 & 0.11 & & \\
\hline PTH, pmol/L & -0.062 & 0.11 & & \\
\hline Venous pH & 0.017 & 0.66 & & \\
\hline Venous $\mathrm{HCO}_{3}{ }^{-}, \mathrm{mmol} / \mathrm{L}$ & 0.106 & 0.007 & & \\
\hline $\mathrm{hsCRP}, \mathrm{mg} / \mathrm{L}$ & -0.167 & $<0.001$ & & \\
\hline NT-pro-BNP, ng/L & -0.387 & $<0.001$ & -0.228 & $<0.001$ \\
\hline Alkaline phosphatase, $\mathrm{U} / \mathrm{L}$ & -0.064 & 0.09 & & \\
\hline Total cholesterol, $\mathrm{mmol} / \mathrm{L}$ & -0.172 & $<0.001$ & & \\
\hline $\mathrm{HDL}$ cholesterol, mmol/L & -0.062 & 0.10 & & \\
\hline LDL cholesterol, $\mathrm{mmol} / \mathrm{L}$ & -0.080 & 0.04 & & \\
\hline Triglycerides, mmol/L & -0.142 & $<0.001$ & & \\
\hline $\begin{array}{l}\text { Carbonylation, } \mathrm{nmol} / \mathrm{mg} \\
\text { protein }\end{array}$ & -0.057 & 0.13 & & \\
\hline Albuminuria, $\mathrm{mg} / 24 \mathrm{~h}$ & -0.123 & 0.001 & & \\
\hline Urinary thiosulfate, $\mu \mathrm{mol} / 24 \mathrm{~h}$ & 0.179 & $<0.001$ & & \\
\hline Urinary sulfate, $\mathrm{mmol} / 24$ & 0.050 & 0.19 & & \\
\hline \multicolumn{5}{|l|}{ Medication } \\
\hline Anti-hypertensives & -0.136 & $<0.001$ & & \\
\hline Statins & -0.114 & 0.003 & & \\
\hline Calcium supplements & -0.042 & 0.27 & & \\
\hline Vitamin D supplements & -0.049 & 0.20 & & \\
\hline Vitamin $\mathrm{K}$ antagonists & -0.139 & $<0.001$ & & \\
\hline Prednisone, mg/d & -0.006 & 0.88 & & \\
\hline Calcineurin inihibitors & -0.160 & $<0.001$ & & \\
\hline Proliferation inhibitor & 0.100 & 0.008 & & \\
\hline Sirolimus & -0.078 & 0.05 & & \\
\hline \multicolumn{5}{|c|}{$\begin{array}{l}\text { Data are presented as standardized beta coefficient (B) with corresponding } P \text {-value. Bold indicates statistical } \\
\text { significance }(P<0.05) \text {. } \\
\text { Abbrevations: } B M I \text {, body mass index; eGFR, estimated glomerular filtration rate; } H b A 1 c \text {, glycated haemoglobin; } \mathrm{HCO}_{3}-\text {, } \\
\text { bicarbonate; } \mathrm{HDL} \text {, high-density lipoprotein; } H L A \text {, human leukocyte antigen; hSCRP, high-sensitivity C-reactive protein; } \\
K T x, \text { kidney transplantation; } L D L \text {, low-density lipoprotein; NT-pro-BNP, N-terminal pro-hormone of brain natriuretic } \\
\text { peptide; PTH, parathyroid hormone; } R-S H \text {, free thiol groups. . }\end{array}$} \\
\hline
\end{tabular}


Median follow-up was 3.1 [2.7 - 3.9] years. During this follow-up, 79 (11\%) RTR died. In the highest tertile of serum R-SH, 16 out of 232 (7\%) died, whereas in the middle tertile 29 out of $230(13 \%)$ and in the lowest tertile 34 out of $233(15 \%)$ died (log-rank test $P=0.003$, Figure 2).

The results of Cox regression analyses for the association of R-SH with all-cause mortality and corresponding Harrell C-indices are shown in Table 3. In Cox regression analysis, a higher level of RSH was significantly associated with a lower mortality risk (HR 0.58 [95\% $\mathrm{Cl} 0.45-0.75$ ], $P<0.001$ per SD increase). After adjustment for age and gender, the association of R-SH with all-cause mortality remained significant (HR 0.64 [95\% $\mathrm{Cl} 0.49-0.84], P=0.001$ per SD increase (model 2)). Upon addition of the other potential confounders of R-SH (model 3), the association of R-SH with all-cause mortality remained significant (HR 0.69 [95\% Cl 0.53-0.93], $P=0.01$ per SD increase (model 3). Additional adjustment for NT-pro-BNP (model 4) resulted in a non-significant hazard ratio for allcause mortality (HR 0.77 [95\% $\mathrm{Cl} 0.57-1.03], P=0.08$ ) indicating a shared causal pathway with R-SH. We found an optimal R-SH cut-off at $1.84 \mathrm{uM} / \mathrm{g}$ (sensitivity 0.72 , specificity 0.50 ) for prediction of allcause mortality. Patients with an R-SH value above $1.84 \mathrm{uM} / \mathrm{g}$ had a reduced risk of all-cause mortality compared with those below $1.84 \mathrm{uM} / \mathrm{g}$ ( $\mathrm{HR} 0.35$ [95\% $\mathrm{Cl} 0.21,0.58], \mathrm{P}<0.001)$ in the unadjusted model.

\section{Fig 2.}

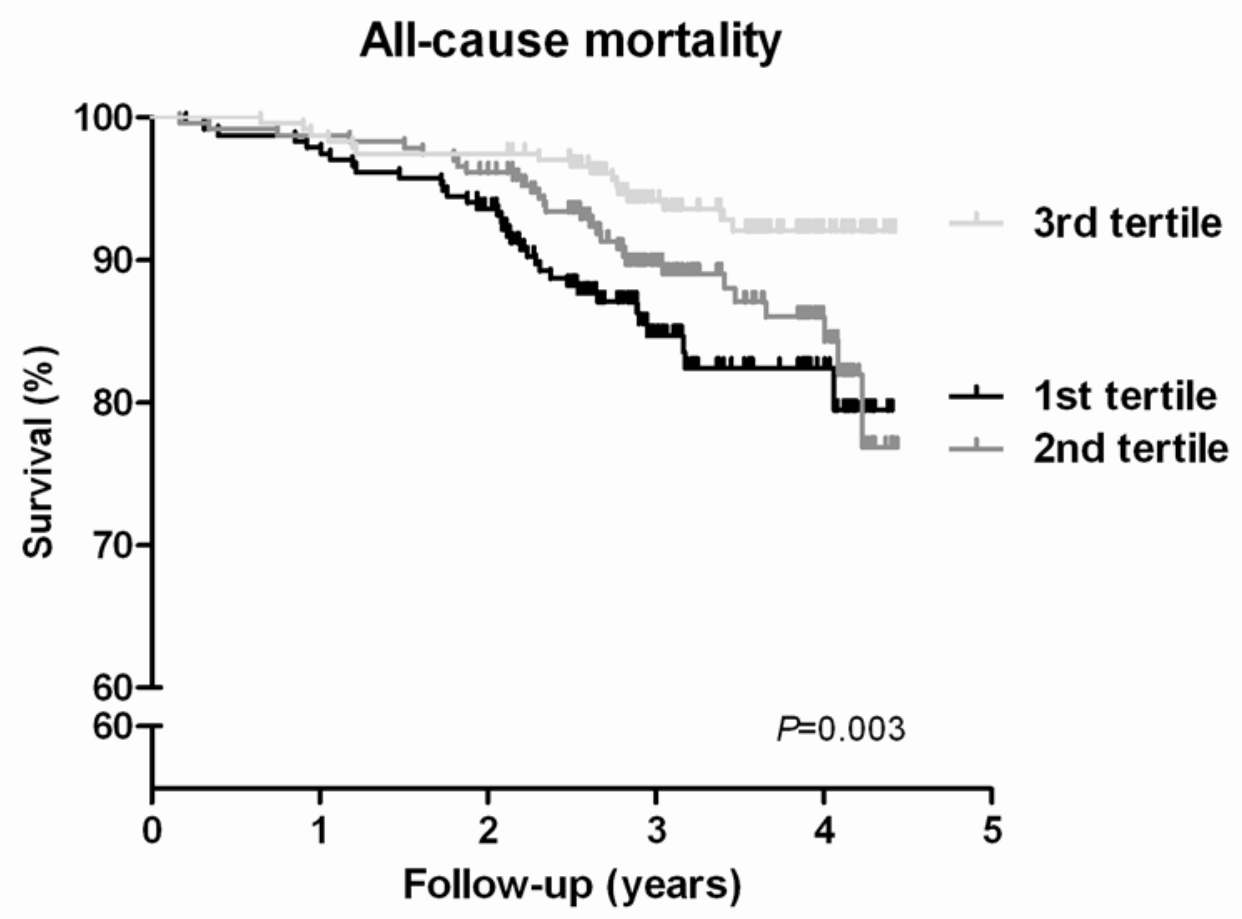

Figure 2. Kaplan-Meier analysis of the association of tertiles of serum R-SH with all-cause mortality Higher serum R-SH is associated with significant survival benefit in renal transplant recipients. Kaplan-Meier curve displayed for patient survival, with log-rank test $P=0.003$. 
Fig 3.

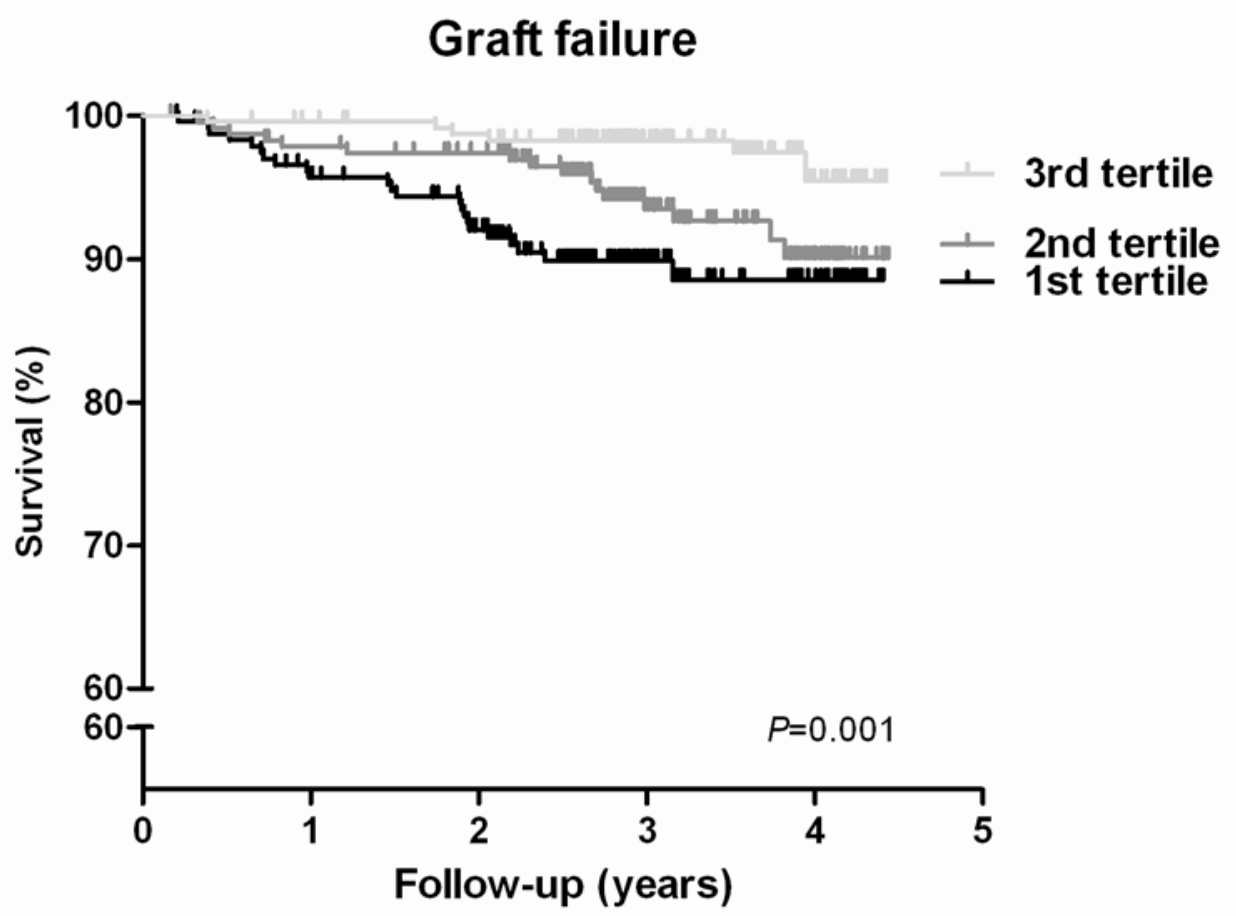

Figure 3. Kaplan-Meier analysis of the association of tertiles of serum R-SH with graft failure

Higher serum R-SH is associated with significant better graft survival in renal transplant recipients.

Kaplan-Meier curve displayed for graft survival, with log-rank test $P=0.001$.

Table 4. Associations of R-SH with graft failure in RTR

\begin{tabular}{|c|c|c|c|}
\hline \multicolumn{4}{|c|}{ Serum R-SH (continuous) } \\
\hline & $\mathrm{HR}(95 \% \mathrm{Cl})$ per SD & $P$-value & Harrell C index \\
\hline Model 1 & $0.42(0.30-0.59)$ & $<0.001$ & 0.704 \\
\hline Model 2 & $0.34(0.24-0.49)$ & $<0.001$ & 0.736 \\
\hline Model 3 & $0.52(0.35-0.78)$ & 0.001 & 0.878 \\
\hline Model 4 & $0.72(0.48-1.10)$ & 0.13 & 0.904 \\
\hline \multicolumn{4}{|c|}{ Model 1: crude } \\
\hline \multicolumn{4}{|c|}{ Model 2: adjusted for age and gender } \\
\hline \multicolumn{4}{|c|}{$\begin{array}{l}\text { Model 3: adjusted for model } 2 \text { plus BMI, HbA1c, eGFR, corrected } \\
\text { calcium, serum TS }\end{array}$} \\
\hline \multicolumn{4}{|c|}{ Model 4: adjusted for model 3 plus NT-pro-BNP } \\
\hline \multicolumn{4}{|c|}{$\begin{array}{l}\text { Abbreviations: } C l \text {, confidence interval; eGFR, estimated glomerular } \\
\text { filtration rate; } H b A 1 c \text {, glycated hemoglobin; HR, hazard ratio; NT- } \\
\text { pro-BNP, N-terminal of the prohormone brain natriuretic peptide } \\
\text { Ref, referent; SD, standard deviation; } R-S H \text {, free thiol groups; TS, } \\
\text { thiosulfate. }\end{array}$} \\
\hline
\end{tabular}




\section{Discussion}

High levels of free serum sulfhydryl groups as a measure of a favorable redox status are associated with a beneficial cardiovascular risk profile and improved patient and graft survival in stable RTR. These associations of R-SH were robust and remained stable also after correcting for multiple potential confounders. To the best of our knowledge, our data are the first to connect total serum free sulfhydryl status with mortality and graft failure risk.

Free sulfhydryl groups (or reduced thiols) are the transducers of redox-regulated events ${ }^{16}$, an area of research that began with the concept of "oxidative stress" involved in cell/organ damage ${ }^{17}$ and has evolved into what is today known as "redox regulation". ${ }^{17}$ An earlier overly simplistic interpretation of the "oxidative stress" concept has led to the unfortunate misconception that all production of ROS is bad and that supplementation with antioxidants is good. However, ROS are now known to be part of elaborate redox signalling networks of considerable spatiotemporal heterogeneity ${ }^{7}$ and antioxidant supplementation has been shown to increase rather than reduce allcause mortality. ${ }^{18}$ Progress in the fledgling field of "redox biology/medicine" is hampered by an incomplete understanding of the relative significance of compartmentalised thiol chemistry in concert with other low molecular weight anti-oxidants and signalling pathways. While there is a general understanding about the relative "pecking order" of antioxidants in biology, based on their fundamental physicochemical oxidation-reduction properties ${ }^{19}$, an integrative understanding of their cross-talk in vivo is still in its infancy. ${ }^{7}$ Much emphasis has been placed on the role of low molecular anti-oxidants such as ascorbate and glutathione, but a key player in the first-line of defense of free radical-related chemistry is nitric oxide ${ }^{20}$, which together with ROS determines cellular redox tone. ${ }^{21}$ NO/ROS balance is linked to thiol redox status (i.e. how much reduced vs oxidized thiols are available) and this is linked to the circulating pool of free thiols in blood. The latter is composed of multiple couples of reduced/oxidized thiols including glutathione, cysteine, homocysteine and also the amounts of free sulfhydryl groups of serum proteins (the majority of which belong to the single sulfhydryl group of serum albumin). Interestingly, the redox status of the glutathione pool has been shown to change with age and lifestyle. ${ }^{22}$

In the present study the levels of total free sulfhydryl groups were measured for two reasons: i) a determination of reduced and oxidized thiols of all of these redox pairs was well beyond the aim of the present study, and ii) to test the hypothesis that protein thiol availability in human serum may be a simple integrative marker of whole-body redox status, which is associated with patient and graft outcomes. Our results show that increased levels of free sulfhydryl groups are beneficially associated with cardiovascular parameters, graft and patient survival in RTR. By serving as a buffer to ROS, R-SH may contribute to protection of graft and patient survival in RTR. ${ }^{23}$ Furthermore it is conceivable that free sulfhydryl groups or reduced thiol availability is linked to NO production. ${ }^{24}$ We propose that higher bodily NO production might have a thiol-sparing effect, which could serve to ensure proper 
functioning of the redox signal transduction network. Whether RTR with higher levels of free sulfhydryl groups indeed have increased bodily NO production needs to be investigated.

Our multivariable analysis revealed that the strongest correlates for $\mathrm{R}$-SH were male gender, eGFR and serum thiosulfate (positively) as well as BMI, HbA1c, corrected calcium and NT-pro-BNP (negatively). The nature of these associations and the effects of R-SH on all these parameters need to be further explored. However, most of the strongest correlates of R-SH are known to influence patient and graft survival post kidney transplantation, since eGFR ${ }^{25,26}$ is favorably associated with patient and graft survival whereas diabetes ${ }^{27,28}$, increased calcium levels ${ }^{29,30}$ and high levels of NTpro-BNP ${ }^{31}$ are associated with worse graft survival and patient outcome in RTR.

In the present study, serum R-SH was strongly inversely associated with NT-pro-BNP, which is a marker of volume overload and heart failure. Furthermore, the association of R-SH with patient and graft survival was lost when NT-pro-BNP was added to the Cox regression analysis. This indicates that R-SH and NT-pro-BNP levels, as a marker of cardiac dysfunction or volume status, might share a common pathway to influence survival in RTR.

Our study has several strengths and limitations. Since it has a cross-sectional design, it is not possible to prove causality between R-SH status and outcomes. Furthermore, our RTR cohort is from a single center and contains almost only Caucasians, limiting generalization of our data. Strengths of our study include the cohort size, relatively long follow-up, a considerable diversity in vintage of the RTR and the extensive clinical and biochemical characterization of our patients.

Our finding that $\mathrm{R}-\mathrm{SH}$ is strongly and independently associated with patient and graft survival raises the question whether and how it could be applied in clinical practice. The hazard ratios of 0.35 and 0.21 at the optimal cut-offs of $1.84 \mathrm{uM} / \mathrm{g}$ and $1.97 \mathrm{uM} / \mathrm{g}$ for mortality and graft failure respectively, indicate that RTR with circulating $\mathrm{R}$-SH concentrations higher than the cut-off have an approximately three times lower future risk of premature death and an approximately five times lower future risk of graft failure. The high relative risk for graft failure, the relatively high sensitivity of 0.87 , the relatively low costs at which the test can be performed (estimated at less than $\$ 2$ per assay), make it a potentially interesting screening tool for early detection of RTR at high risk for future development of graft failure. The relatively high sensitivity of 0.87 indicates that there will be a low rate of false negatives. The low specificity of 0.39 indicates that there will be a high rate of false positives, which precludes the use of assessment of R-SH as a diagnostic tool, but rather only as a screening tool, which seems logical from the perspective that oxidative stress is likely involved in the development of graft failure, but related to much more pathophysiological processes than that of graft failure alone. Whether or not it can be used as an initial screening tool would depend on circumstances, which can change over time. If a positive result would require invasive, costly and risky further testing (e.g. renal biopsy), the relatively high rate of false positivity might be a problem. If, however, developments would proceed and a positive result could guide further non-invasive 
testing, e.g. assessment of an miRNA profile, the relatively high risk of false positivity might be acceptable. The same would be true if there were a simple, cheap, non-toxic treatment or change in diet or lifestyle, that would raise circulating R-SH concentrations and decrease risk of graft failure (and mortality). In that circumstance it might be a screening test that could be applied without necessity of further testing.

In conclusion, serum R-SH is associated with improved patient and graft survival in RTR after adjustment for potential confounders. The association was weakened after additional adjustment for NT-pro-BNP, suggesting that the beneficial effects of R-SH on cardiorenal outcomes may be mediated by volume status. Interventions that regulate the extracellular redox status may be effective in preventing cardiovascular morbidity and mortality in RTR. Last but not least, our study suggests that simple measurement of total serum thiol status may be indicative of whole-body oxidative stress and a simple yet potentially predictive tool for future translational studies. This would merit further testing including direct comparison with established markers of oxidative stress such as 8isoprostanes and 4-hydroxynonenal in larger cohorts.

\section{Acknowledgements}

The authors would like to express their gratitude towards Beatrix Blanchard for her expertise and valuable help in measuring serum free sulfhydryl groups and thiosulfate in serum and urine. This work was supported by Grants from the Dutch Kidney Foundation (NSN C08-2254, P13-114), COST Action BM1005: ENOG: European Network on Gasotransmitters (www.gasotransmitters.eu), and the Dutch Top Institute Food and Nutrition (grants A-1003 and CH-003).

\section{References}

1. Meier-Kriesche HU, Schold JD, Srinivas TR, et al. Kidney transplantation halts cardiovascular disease progression in patients with end-stage renal disease. Am J Transplant 2004; 4: 1662-1668.

2. Jardine AG, Gaston RS, Fellstrom BC, Holdaas H. Prevention of cardiovascular disease in adult recipients of kidney transplants. Lancet 2011; 378: 1419-1427.

3. La Manna G, Lanci N, Della Bella E, et al. Reduction of oxidative damage reflects a better kidney transplantation outcome. Am J Nephrol 2011; 34: 1303-1312.

4. Mangge H, Becker K, Fuchs D, et al. Antioxidants, inflammation and cardiovascular disease. World J Cardiol 2014; 6: 462-477.

5.Banne AF, Amiri A, Pero RW. Reduced levels of serum thiols in patients with a diagnosis of active disease. J Anti Aging Med 2003; 6: 327-334. 
6. Go YM, Jones DP. Cysteine/cystine redox signaling in cardiovascular disease. Free Radic Biol Med 2011; 50: 495-509.

7. Jones DP, Sies H. The Redox Code. Antioxid Redox Signal 2015; 23:734-46.

8. van den Berg E, Pasch A, Westendorp WH, et al. Urinary sulfur metabolites associate with a favorable cardiovascular risk profile and survival benefit in renal transplant recipients. J Am Soc Nephrol 2014; 25: 1303-1312.

9. van den Berg E, Engberink MF, Brink EJ, et al. Dietary acid load and metabolic acidosis in renal transplant recipients. Clin J Am Soc Nephrol 2012; 7: 1811-1818.

10. van den Berg E, Geleijnse JM, Brink EJ, et al. Sodium intake and blood pressure in renal transplant recipients. Nephrol Dial Transplant 2012; 27: 3352-3359.

11. Farese S, Stauffer E, Kalicki R, et al. Sodium thiosulfate pharmacokinetics in hemodialysis patients and healthy volunteers. Clin J Am Soc Nephrol 2011; 6: 1447-1455.

12. Levey $A S$, Stevens $L A$, Schmid $C H$, et al. A new equation to estimate glomerular filtration rate. Ann Intern Med 2009; 150: 604-612.

13. Levine RL, Garland D, Oliver CN, et al. Determination of carbonyl content in oxidatively modified proteins. Methods Enzymol 1990; 186: 464-478.

14. Ellman GL. Tissue sulfhydryl groups. Arch Biochem Biophys 1959; 82: 70-77.

15. Hu ML, Louie S, Cross CE, et al. Antioxidant protection against hypochlorous acid in human plasma. J Lab Clin Med 1993; 121: 257-262.

16. Cooper CE, Patel RP, Brookes PS, et al. Nanotransducers in cellular redox signaling: modification of thiols by reactive oxygen and nitrogen species. Trends Biochem Sci 2002; 27: 489-492.

17. Sies H. Oxidative stress: a concept in redox biology and medicine. Redox Biol 2015; 4: 180-183.

18. Bjelakovic G, Nikolova D, Gluud LL, et al. Antioxidant supplements for prevention of mortality in healthy participants and patients with various diseases. Cochrane Database Syst Rev 2008; 2: CD007176.

19. Buettner GR. The pecking order of free radicals and antioxidants: lipid peroxidation, alphatocopherol, and ascorbate. Arch Biochem Biophys 1993; 300: 535-543.

20. Wink DA, Miranda KM, Espey MG, et al. Mechanisms of the antioxidant effects of nitric oxide. Antioxid Redox Signal 2001; 3: 203-213.

21. Landar A, Darley-Usmar VM. Nitric oxide and cell signaling: modulation of redox tone and protein modification. Amino Acids 2003; 25: 313-321.

22. Jones, Mody VC Jr, Carlson, et al. Redox analysis of human plasma allows separation of prooxidant event of aging from decline in antioxidant defenses. Free Radic Biol Med 2002; 33: 12901300.

23. Bryan NS, Rassaf T, Maloney RE, et al. Cellular targets and mechanisms of nitros(yl)ation: an insight into their nature and kinetics in vivo. Proc Natl Acad Sci USA 2004; 101: 4308-4313. 
24. Murphy ME, Piper HM, Watanabe $H$, et al. Nitric oxide production by cultured aortic endothelial cells in response to thiol depletion and replenishment. J Biol Chem 1991; 266: 19378-19383.

25. Smith-Palmer J, Kalsekar A, Valentine W. Influence of renal function on long-term graft survival and patient survival in renal transplant recipients. Curr Med Res Opin 2014; 30: 235-242.

26. Soveri I, Holdaas $\mathrm{H}$, Jardine $\mathrm{A}$, et al. Renal transplant dysfunction--importance quantified in comparison with traditional risk factors for cardiovascular disease and mortality. Nephrol Dial Transplant 2006; 21: 2282-2289.

27. Gill JS, Abichandani R, Kausz AT, Pereira BJ. Mortality after kidney transplant failure: the impact of non-immunologic factors. Kidney Int 2002; 62: 1875-1883.

28. Cosio FG, Pesavento TE, Kim S, et al. Patient survival after renal transplantation: IV. Impact of post-transplant diabetes. Kidney Int 2002; 62: 1440-1446.

29. Moore J, Tomson CR, Tessa Savage M, et al. Serum phosphate and calcium concentrations are associated with reduced patient survival following kidney transplantation. Clin Transplant 2011; 25: 406-416.

30. Mehrens T, Thiele S, Suwelack B, et al. The beneficial effects of calcium channel blockers on longterm kidney transplant survival are independent of blood-pressure reduction. Clin Transplant 2000; 14: $257-261$

31. Oterdoom LH, de Vries AP, van Ree RM, et al. N-terminal pro-B-type natriuretic peptide and mortality in renal transplant recipients versus the general population. Transplantation 2009; 87: 1562-1570. 